\title{
Extensions of Borel Measurable Maps and Ranges of Borel Bimeasurable Maps
}

by

\author{
Petr HOLICKÝ
}

\author{
Presented by Czestaw BESSAGA
}

\begin{abstract}
Summary. We prove an abstract version of the Kuratowski extension theorem for Borel measurable maps of a given class. It enables us to deduce and improve its nonseparable version due to Hansell. We also study the ranges of not necessarily injective Borel bimeasurable maps $f$ and show that some control on the relative classes of preimages and images of Borel sets under $f$ enables one to get a bound on the absolute class of the range of $f$. This seems to be of some interest even within separable spaces.
\end{abstract}

1. Introduction. The main aim of this note is to give new results on the absolute Borel classes of the ranges of Borel bimeasurable (not necessarily injective) maps between metric spaces. The main tool is the characterization of spaces of absolute Borel class by means of complete sequences of covers, which was given in [10, Theorem 2.2] and also in [8, Theorems 2.5 and 3.8] for a more general setting.

An abstract version of the Kuratowski extension theorem is proved that can be applied to classes of Borel sets. We improve the results obtained by Hansell for maps between nonseparable metric spaces. Hansell generalized classical theorems of Kuratowski on extension of Borel measurable maps [2, Theorem 9], on extension of Borel isomorphisms [2, Theorem 10], and on absolute Borel classes of images of Borel isomorphisms [2, Theorem 11]. He

2000 Mathematics Subject Classification: 26A21, 28A05, 54H05.

Key words and phrases: absolute Borel sets, Borel classes, Borel isomorphisms, Borel bimeasurable maps, nonseparable spaces, scattered families.

The author was supported by the "Mathematics in Information Society" project carried out by Alfréd Rényi Institute of Mathematics-Hungarian Academy of Sciences, in the framework of the European Community's "Confirming The International Role of Community Research" program. The research was also partly supported by grants GAČR 201/03/0933, GAČR 201/03/0931 and MSM 113200007. 
left open the cases of limit Borel classes. Our method of proof includes the limit cases as well.

We give the corresponding results also for scattered-Borel sets, a family of sets which coincides with the family of extended Borel, or equivalently bi-Suslin, sets in complete metric spaces and which was introduced and studied by Hansell.

We deal with one possible hierarchy of multiplicative and additive classes $\mathcal{M}_{\alpha}^{s}$ and $\mathcal{A}_{\alpha}^{s}$ of scattered-Borel sets. Let us point out that in the mostly studied cases of Borel classes with $\alpha<\omega_{1}$ in metric spaces, the corresponding classes of Borel and scattered-Borel sets coincide. Moreover, the extended hierarchy, which can be arbitrarily long, exhausts all extended Borel sets of Hansell in metric spaces and all scattered-Borel sets in topological spaces. The choice of the hierarchy for $\alpha \geq \omega_{1}$, or of the entire hierarchy in topological spaces, is not the only natural one. However, one may notice that our results give some information also for other natural hierarchies (cf., e.g., Remark 3.4 below).

2. Notation and some basic facts. The families of open, or closed, sets of a topological space $X$ are denoted by $\mathcal{G}=\mathcal{G}(X)$, or $\mathcal{F}=\mathcal{F}(X)$, respectively.

Let $\mathcal{S}$ and $\mathcal{T}$ be families of subsets of $X$. We use $\mathcal{S} \wedge \mathcal{T}$ to denote the family $\{S \cap T: S \in \mathcal{S}, T \in \mathcal{T}\}$.

A pairwise disjoint family $\mathcal{S}$ of subsets of $X$ is scattered if each nonempty subfamily $\mathcal{T} \subset \mathcal{S}$ contains a $T \in \mathcal{T}$ which is relatively open in $\bigcup \mathcal{T}$. We use later without further reference the easy fact (see, e.g., [5]) that $\mathcal{S}$ is scattered if and only if there is a well-ordering of elements of $\mathcal{S}$ such that $\mathcal{S}=\left\{S_{\alpha}: \alpha<\kappa\right\}$ and there are open sets $G_{\alpha}, \alpha<\kappa$, such that the indexed family $\left\{G_{\alpha}: \alpha<\kappa\right\}$ is increasing and $S_{\alpha} \subset G_{\alpha} \backslash \bigcup_{\beta<\alpha} G_{\beta}$ for $\alpha<\kappa$. It follows immediately (see [5, Lemma 2.3]) that if $\mathcal{S}$ is a scattered family, then there is an $\widehat{S} \in \mathcal{F} \wedge \mathcal{G}$ for every $S \in \mathcal{S}$ such that $S \subset \widehat{S}$ and the family $\{\widehat{S}: S \in \mathcal{S}\}$ is scattered.

It is not difficult to check that the family $\mathcal{S} \wedge \mathcal{T}$ is scattered if both $\mathcal{S}$ and $\mathcal{T}$ are scattered families of subsets of a topological space.

The sets which can be described as unions of scattered subfamilies of $\mathcal{F}(X) \wedge \mathcal{G}(X)$ (we also write $(\mathcal{F} \wedge \mathcal{G})(X))$ are called $H$-sets and the family of all $H$-sets in $X$ is denoted by $\mathcal{H}=\mathcal{H}(X)$. It is well known that $\mathcal{H}(X)$ is an algebra (see, e.g., $[9$, Theorem $1, \S 12, \mathrm{VI}]$ ).

The family of all results of the Suslin operation applied to elements of a family $\mathcal{T}$ of subsets of a given set is denoted by $\mathcal{S}(\mathcal{T})$.

The elements of $\mathcal{S}(\mathcal{F}(X))$ are called Suslin and a metric space is absolute Suslin (with respect to metric spaces) if it is Suslin in its completion. 
A Tikhonov space is called absolute $\mathcal{S}(\mathcal{H})$ if it is $\mathcal{S}(\mathcal{H})$ in some compactification, or equivalently, in every Tikhonov space in which it is embedded (see, e.g., $[4,6.14]$ ). It was shown in $[6$, Theorem 3(b)] that absolute $\mathcal{S}(\mathcal{H})$ spaces coincide with the scattered-K-analytic spaces, called originally almost- $K$-descriptive by Hansell who introduced them. Let us recall that metrizable scattered- $K$-analytic spaces are absolute Suslin as every $H$-set is a Suslin set in any metrizable space.

We refer further to the following results on additive families. A family $\mathcal{S}$ is called $\mathcal{L}$-additive if $\bigcup \mathcal{T} \in \mathcal{L}$ for every nonempty $\mathcal{T} \subset \mathcal{S}$.

The family $\mathcal{S}$ is a refinement of $\mathcal{T}$ if $\bigcup \mathcal{S}=\bigcup \mathcal{T}$ and if, for every $S \in \mathcal{S}$, there is a $T \in \mathcal{T}$ such that $S \subset T$.

The family $\mathcal{S}$ is a network of $\mathcal{T}$ if every $T \in \mathcal{T}$ is the union of the family $\{S \in \mathcal{S}: S \subset T\}$.

It was shown in [1, Theorem 2] (taking into account [6, Theorem 3(b)]) that any pairwise disjoint $\mathcal{S}(\mathcal{F})$-additive family in a complete metric space has a $\sigma$-discrete, and thus also a $\sigma$-scattered, network.

It was shown in $[7$, Theorem $2(\mathrm{a})]$ that any pairwise disjoint $\mathcal{S}(\mathcal{H})$ additive family in a compact space has a $\sigma$-scattered network if the cardinality of the family is nonmeasurable (i.e., there is no nontrivial two-valued probability measure on the corresponding cardinal).

We use $\mathcal{B}=\mathcal{B}(X)$ to denote the family of Borel subsets of $X$. The smallest $\sigma$-field containing $\mathcal{B}(X)$ and closed under taking unions of scattered subfamilies is denoted by $\mathcal{B}^{s}=\mathcal{B}^{s}(X)$ here and its elements are called scattered-Borel sets. In a metric space, scattered-Borel sets are just the "extended Borel sets" of [3]. Indeed, the family of extended Borel sets (the smallest $\sigma$-algebra closed under discrete unions) is a subfamily of $\mathcal{B}^{s}$. If $\mathcal{S}$ is a scattered family of extended-Borel sets, then there is a well-ordering of elements of $\mathcal{S}$ such that $\mathcal{S}=\left\{B_{\alpha}: \alpha<\kappa\right\}$ and there are open sets $G_{\alpha}, \alpha<\kappa$, such that the indexed family $\left\{G_{\alpha}: \alpha<\kappa\right\}$ is increasing and $B_{\alpha} \subset G_{\alpha} \backslash \bigcup_{\beta<\alpha} G_{\beta}$ for $\alpha<\kappa$. Let $\mathcal{V}=\bigcup_{n \in \mathbb{N}} \mathcal{V}_{n}$ be a base for the topology of the metric space such that each $\mathcal{V}_{n}$ is discrete. Then the family $\left\{V \cap B_{\alpha}: \alpha<\kappa, n \in \mathbb{N}, V \in \mathcal{V}_{n}, V \subset G_{\alpha}\right\}$ forms a $\sigma$-discrete refinement of $\mathcal{S}$ by extended Borel sets. Hence the family of extended Borel sets is closed under scattered unions and coincides with the family of scattered-Borel sets.

Given a family $\mathcal{L}$ of subsets of $X$, the symbols $\mathcal{L}_{s}, \mathcal{L}_{\sigma}, \mathcal{L}_{\delta}$, and $\mathcal{L}_{c}$ stand for the families of all scattered unions, countable unions, countable intersections, and complements of elements of $\mathcal{L}$, respectively. Note that, e.g., $\mathcal{L}_{s \sigma}$ consists of the unions of all $\sigma$-scattered subfamilies of $\mathcal{L}$.

Let $\mathcal{A}_{\alpha}$ and $\mathcal{M}_{\alpha}, 0 \leq \alpha<\omega_{1}$, stand for the additive and multiplicative classes of Borel sets, respectively. We introduce a hierarchy of scatteredBorel sets. Let $\mathcal{A}_{1}^{s}=\mathcal{H}_{\sigma}$ and $\mathcal{M}_{1}^{s}=\mathcal{H}_{\delta}$. Then we introduce the additive, and multiplicative, classes for $\alpha>1$ by $\mathcal{A}_{\alpha}^{s}=\left(\bigcup_{\beta<\alpha} \mathcal{M}_{\beta}^{s}\right)_{s \sigma}$ and $\mathcal{M}_{\alpha}^{s}=$ 
$\left(\bigcup_{\beta<\alpha} \mathcal{A}_{\beta}^{s}\right)_{\delta}$, respectively. We use here the notation $\mathcal{A}_{0}^{s}=\mathcal{A}_{0}=\mathcal{G}$ and $\mathcal{M}_{0}^{s}=\mathcal{M}_{0}=\mathcal{F}$. In metric spaces, for $1 \leq \alpha<\omega_{1}$, the above additive and multiplicative classes coincide with the standard Borel classes. On the other hand, for $\alpha \geq \omega_{1}$, the classes $\mathcal{M}_{\alpha}^{s}$ and $\mathcal{A}_{\alpha}^{s}$ may not be complementary, which causes some troubles. The reason for this choice of the hierarchy is the possibility to describe the absolute multiplicative classes by complete sequences of covers. For more information see [8].

Let us say that a map $f$ of a topological space $X$ to a topological space $Y$ is (scattered-) Borel measurable of class $\alpha$ if the preimages of sets of additive, or multiplicative, class $\gamma$ are of additive, or multiplicative, class $\alpha+\gamma$, respectively. In metrizable spaces for $\alpha<\omega_{1}$ it is equivalent to say that the preimages of open sets are (scattered-) Borel sets of additive class $\alpha$. If $f$ is a map of $X$ to a topological space $Y$ and $\mathcal{L}$ is a family of subsets of $X$, we say that $f$ is $\mathcal{L}$-measurable if $f^{-1}(\mathcal{G}(Y))\left(=\left\{f^{-1}(G): G \in \mathcal{G}(Y)\right\}\right) \subset \mathcal{L}$. We explain in Lemma 2.3 the relation of this and the previously defined measurability of maps even for higher classes of scattered-Borel sets.

A bijective map $f$ between topological spaces $X$ and $Y$ is called a (scattered-) Borel isomorphism of class $(\alpha, \beta)$ if $f$ is (scattered-) Borel measurable of class $\alpha$ and $f^{-1}$ is (scattered-) Borel measurable of class $\beta$.

Let us sum up a few properties of the introduced hierarchies of scatteredBorel sets that we need in what follows. They are quite straightforward and most of them can be found, e.g., in [8, Lemma 1.5].

LEMma 2.1. (a) The family of all scattered-Borel sets in a space can be described by the additive and multiplicative classes as follows:

$$
\mathcal{B}^{s}=\bigcup_{\alpha<\kappa} \mathcal{A}_{\alpha}^{s}=\bigcup_{\alpha<\kappa} \mathcal{M}_{\alpha}^{s}
$$

for sufficiently large ordinals $\kappa$.

(b) The classes $\mathcal{A}_{\alpha}^{s}$ and $\mathcal{M}_{\alpha}^{s}$ are closed under finite unions and finite intersections.

(c) The following relations hold:

$$
\begin{gathered}
\mathcal{M}_{\alpha}^{s} \cup \mathcal{A}_{\alpha}^{s} \subset \mathcal{M}_{\alpha+1}^{s} \cap \mathcal{A}_{\alpha+1}^{s}, \quad \mathcal{A}_{\alpha c}^{s} \subset \mathcal{M}_{\alpha+1}^{s} \quad \text { for } \alpha \geq 0, \\
\mathcal{M}_{\alpha c}^{s} \subset \mathcal{M}_{\alpha \sigma}^{s} \subset \mathcal{A}_{\alpha+1}^{s} \quad \text { for } \alpha>0 .
\end{gathered}
$$

(d) The classes $\mathcal{A}_{\alpha}^{s}$ are closed under scattered unions for $\alpha>0$.

(e) The classes $\mathcal{M}_{\alpha}^{s}$ are closed under scattered unions if $\alpha>0$ is nonlimit or if $\alpha \in\left(0, \omega_{1}\right)$.

(f) If $\alpha<\omega_{1}$, then $\mathcal{A}_{\alpha c}^{s}=\mathcal{M}_{\alpha}^{s}$.

Proof. The inclusion $\mathcal{M}_{\alpha c}^{s} \subset \mathcal{M}_{\alpha \sigma}^{s}$ in (c) was not stated in [8] explicitly. 
Notice however that

$$
\mathcal{M}_{\alpha c}^{s}=\left(\bigcup_{\beta<\alpha} \mathcal{A}_{\beta}^{s}\right)_{\delta c}=\left(\bigcup_{\beta<\alpha} \mathcal{A}_{\beta c}^{s}\right)_{\sigma} \subset \mathcal{M}_{\alpha \sigma}^{s}
$$

since $\mathcal{A}_{\beta c}^{s} \subset \mathcal{M}_{\beta+1}^{s} \subset \mathcal{M}_{\alpha}^{s}$ for $\beta<\alpha$.

To verify (e), let $M_{a}=\bigcap_{n \in \mathbb{N}} A_{n}^{a}, a \in I$, for some $A_{n}^{a} \in \mathcal{A}_{\beta_{n}}^{s}, 1 \leq \beta_{n}<$ $\alpha<\omega_{1}$, or $A_{n}^{a} \in \mathcal{H}$, and let the family $\left\{M_{a}: a \in I\right\}$ be scattered. Then

$$
\bigcup_{a \in I} M_{a}=\bigcap_{n \in \mathbb{N}} \bigcup_{\beta<\alpha} \bigcup\left\{A_{n}^{a} \cap \widehat{M}_{a}: A_{n}^{a} \in \mathcal{A}_{\beta}^{s}\right\} .
$$

Here $\left\{\widehat{M}_{a}: a \in I\right\}$ is a scattered family of elements of $\mathcal{F} \wedge \mathcal{G}$ with $M_{a} \subset \widehat{M}_{a}$ for $a \in I$. Using the fact that the additive classes are closed under both scattered and countable unions, we deduce that $\bigcup_{a \in I} M_{a} \in \mathcal{M}_{\alpha}^{s}$.

If $\alpha$ is nonlimit, the argument is still simpler as we may use a single $\beta<\alpha$ in the description, namely the one with $\beta+1=\alpha$.

It remains to prove (f). The equalities $\mathcal{A}_{0 c}^{s}=\mathcal{M}_{0}^{s}$ and $\mathcal{A}_{1 c}^{s}=\mathcal{M}_{1}^{s}$ follow easily from the definitions and the fact that $\mathcal{H}$ is an algebra. If $\alpha \in\left(1, \omega_{1}\right)$, then

$$
\mathcal{M}_{\alpha}^{s}=\left(\bigcup_{\beta<\alpha} \mathcal{A}_{\beta}^{s}\right)_{\delta}=\left(\bigcup_{\beta<\alpha} \mathcal{M}_{\beta c}^{s}\right)_{\delta}=\left(\bigcup_{\beta<\alpha} \mathcal{M}_{\beta}^{s}\right)_{\sigma c}=\mathcal{A}_{\alpha c}^{s}
$$

where we used the induction hypothesis and the just proved fact that $\mathcal{M}_{\beta}^{s}$ is closed under scattered unions for $\beta<\omega_{1}$.

We need a lemma on measurability of $\sigma$-scattered refinements:

LEMMA 2.2. Let $\mathcal{L}$ be a family of subsets of a topological space $X$ which is closed under finite intersections and contains $(\mathcal{F} \wedge \mathcal{G})(X)$. Let $\mathcal{S} \subset \mathcal{L}$ have a $\sigma$-scattered refinement. Then there is a $\sigma$-scattered refinement $\mathcal{T}$ of $\mathcal{S}$ with $\mathcal{T} \subset \mathcal{L}$.

Proof. Let $\mathcal{R}=\bigcup_{n \in \mathbb{N}} \mathcal{R}_{n}$ be a refinement of $\mathcal{S}$ with each $\mathcal{R}_{n}$ scattered. We find for each $n \in \mathbb{N}$ and $R \in \mathcal{R}_{n}$ a set $\widehat{R} \in(\mathcal{F} \wedge \mathcal{G})(X)$ such that the family $\left\{\widehat{R}: R \in \mathcal{R}_{n}\right\}$ is still scattered and $R \subset \widehat{R}$ for $R \in \mathcal{R}_{n}$. Fix now for every $R \in \mathcal{R}$ one choice of an $S(R) \in \mathcal{S}$ such that $R \subset S(R)$ and put $\mathcal{T}=\{\widehat{R} \cap S(R): R \in \mathcal{R}\}$. The family $\mathcal{T}$ is $\sigma$-scattered because $\bigcup_{n \in \mathbb{N}}\left\{\widehat{R}: R \in \mathcal{R}_{n}\right\}$ is $\sigma$-scattered. Since $\bigcup \mathcal{R}=\bigcup \mathcal{S}$ and $\bigcup \mathcal{R} \subset \bigcup \mathcal{T} \subset \bigcup \mathcal{S}$, we have $\bigcup \mathcal{T}=\bigcup \mathcal{S}$, and finally, $\mathcal{T}$ is a refinement of $\mathcal{S}$ since each $T \in \mathcal{T}$ is of the form $T=\widehat{R} \cap S(R) \subset S(R)$ for an $S(R) \in \mathcal{S}$.

The classes of scattered-Borel sets are produced, besides the set-theoretical operations of countable union and countable intersection, by using the topological operation of taking unions of scattered families, but $f^{-1}(\mathcal{S})$ need not be scattered for scattered families $\mathcal{S}$ unless some additional assumptions on $f$ are imposed. However, we get the following statement. 
Lemma 2.3. (a) Let $f$ be an $\mathcal{A}_{\alpha}^{s}$-measurable map of a topological space $X$ to a metric space $M$ and let the family $f^{-1}(\mathcal{G}(M))$ have a $\sigma$ scattered network. Then $f^{-1}(\mathcal{S})$ has a $\sigma$-scattered network for every scattered family $\mathcal{S}$ in $M$ and $f$ is scattered-Borel measurable of class $\alpha+1$. If $\alpha<\omega_{1}$, then $f$ is of class $\alpha$.

(b) Let $f: X \rightarrow Y$ be a map between topological spaces such that $f^{-1}(\mathcal{S})$ has a $\sigma$-scattered network for every scattered family $\mathcal{S}$ in $Y$, and suppose that $f$ is $\mathcal{A}_{\alpha}^{s}$-measurable. Then $f$ is scattered-Borel measurable of class $\alpha+3$. If moreover $\alpha<\omega_{1}$, then $f$ is of class $\alpha$.

Proof. (a) Let $\mathcal{S}$ be any scattered family in $M$. Then there is some well-ordering of elements of $\mathcal{S}$ such that $\mathcal{S}=\left\{S_{\alpha}: \alpha<\kappa\right\}$ and there is an increasing family of open sets $G_{\alpha}, \alpha<\kappa$, such that $S_{\alpha} \subset G_{\alpha} \backslash \bigcup_{\beta<\alpha} G_{\beta}$. Let $\mathcal{V}=\bigcup_{n \in \mathbb{N}} \mathcal{V}_{n}$ be a network of $f^{-1}(\mathcal{G}(M))$ such that each $\mathcal{V}_{n}$ is scattered. It is not difficult to verify that the family $\left\{f^{-1}\left(S_{\alpha}\right) \cap V: \alpha<\kappa, n \in \mathbb{N}\right.$, $\left.V \in \mathcal{V}_{n}, V \subset f^{-1}\left(G_{\alpha}\right)\right\}$ is a $\sigma$-scattered network of $\left\{f^{-1}(S): S \in \mathcal{S}\right\}$. Notice that for each $V \in \mathcal{V}$ the intersection $f^{-1}\left(S_{\alpha}\right) \cap V$ is nonempty for at most one $\alpha$.

Also $f^{-1}(\mathcal{F}) \subset \mathcal{A}_{\alpha c}^{s} \subset \mathcal{M}_{\alpha+1}^{s}$, and if $\alpha<\omega_{1}$, then $\mathcal{A}_{\alpha c}^{s}=\mathcal{M}_{\alpha}^{s}$ by Lemma 2.1(f).

Proceeding now by transfinite induction we prove that preimages of sets of additive, or multiplicative, class $\gamma$ are of class $\alpha+1+\gamma$ (and $\alpha+\gamma$ if $\left.\alpha<\omega_{1}\right)$. When investigating the unions of preimages of elements of a scattered family of sets of a given multiplicative class $\gamma$, we use the existence of a $\sigma$-scattered network which we may find by the induction hypothesis and by Lemma 2.2 in the corresponding multiplicative class $\alpha+1+\gamma$.

(b) The elements of $f^{-1}\left(\mathcal{A}_{1}^{s}\right)$ belong to $\left(\mathcal{A}_{\alpha}^{s} \wedge \mathcal{A}_{\alpha c}^{s}\right)_{s \sigma} \subset \mathcal{A}_{\alpha+2}^{s}$, and those of $f^{-1}\left(\mathcal{M}_{1}^{s}\right)$ to $\left(\mathcal{A}_{\alpha+2}^{s}\right)_{c} \subset \mathcal{M}_{\alpha+3}^{s}$. If $\alpha<\omega_{1}$ then we have $\left(\mathcal{A}_{\alpha}^{s} \cap \mathcal{A}_{\alpha c}^{s}\right)_{s \sigma}=$ $\left(\mathcal{A}_{\alpha}^{s} \cap \mathcal{M}_{\alpha}^{s}\right)_{s \sigma} \subset \mathcal{A}_{\alpha+1}^{s}$ and also $\left(\mathcal{A}_{\alpha+1}^{s}\right)_{c}=\mathcal{M}_{\alpha+1}^{s}$ by Lemma 2.1(f) again. We conclude the proof by induction as in (a).

We now state a lemma on the graph of a measurable map. The main examples of the families $\mathcal{A}$ and $\mathcal{A}^{*}$ are the additive classes of (scattered-) Borel sets in $X$ and in $X \times M$, respectively.

Lemma 2.4. Let $\mathcal{A}$ be a family of subsets of a topological space $X$ which contains $(\mathcal{F} \wedge \mathcal{G})(X)$ and which is closed under finite intersections and $\sigma$ scattered unions. Assume that a map $f$ of $X$ to a metric space $(M, \varrho)$ is $\mathcal{A}$-measurable and that $f^{-1}(\mathcal{G}(M))$ has a $\sigma$-scattered network.

(a) Setting $g: x \mapsto(x, f(x))$ we have $g^{-1}(\mathcal{G}(X \times M)) \subset \mathcal{A}$ and the family $g^{-1}(\mathcal{S})$ has a $\sigma$-scattered network for every scattered family $\mathcal{S}$ in $X \times M$. 
(b) The graph of $f$ belongs to $\mathcal{A}_{c}^{*} \cap \mathcal{A}_{\delta}^{*}$, where $\mathcal{A}^{*}$ is the smallest family containing $\mathcal{A} \times \mathcal{G}(M)$ and $(\mathcal{F} \wedge \mathcal{G})(X \times M)$, closed under finite intersections and $\sigma$-scattered unions.

Proof. (a) Let $\mathcal{V}=\bigcup_{n \in \mathbb{N}} \mathcal{V}_{n}$ be a base of the topology of $M$ such that each $\mathcal{V}_{n}$ is discrete (and thus pairwise disjoint).

Let $\mathcal{N}_{n}$ be a $\sigma$-scattered network of $f^{-1}\left(\mathcal{V}_{n}\right)$ consisting of elements of $\mathcal{A}$, which exists by Lemma 2.2. Now $\mathcal{N}=\bigcup_{n \in \mathbb{N}} \mathcal{N}_{n}$ is a $\sigma$-scattered network for $f^{-1}(\mathcal{G}(M))$ by sets from $\mathcal{A}$.

Every open set $G \subset X \times M$ equals $\bigcup_{V \in \mathcal{V}}\left(U_{V} \times V\right)$, where $U_{V}$ is the maximal open set such that $U_{V} \times V \subset G$. Then

$$
g^{-1}(G)=\bigcup_{n \in \mathbb{N}} \bigcup_{V \in \mathcal{V}_{n}} \bigcup_{N \in \mathcal{N}_{V}}\left(U_{V} \cap N\right)
$$

where $\mathcal{N}_{V}=\left\{N \in \mathcal{N}: N \subset f^{-1}(V)\right\}$. Note that the families $\mathcal{N}_{V} \subset \mathcal{N}$ that correspond to distinct elements $V$ of $\mathcal{V}_{n}$ for a fixed $n \in \mathbb{N}$ are disjoint. It follows that $g^{-1}(G) \in \mathcal{A}_{s \sigma}=\mathcal{A}$ as it is the union of a $\sigma$-scattered family of elements of $\mathcal{A}$.

It remains to investigate $g^{-1}(\mathcal{S})$ for $\mathcal{S}$ scattered in $X \times M$. Without loss of generality we may suppose that $\mathcal{S}$ is the family of differences $S_{\alpha}=$ $G_{\alpha} \backslash \bigcup_{\beta<\alpha} G_{\beta}, \alpha<\kappa$, for some increasing family of open sets (indexed by ordinals $\alpha<\kappa)$. Let $U_{V}^{\alpha}$ be the maximal open set such that $U_{V}^{\alpha} \times V \subset G_{\alpha}$ for $V \in \mathcal{V}$. Then the family of $\left(U_{V}^{\alpha} \times V\right) \cap S_{\alpha}$ with $V \in \mathcal{V}$ is a refinement of $\mathcal{S}$. It is now sufficient to prove that for every $n \in \mathbb{N}$ the family

$$
\left\{g^{-1}\left(\left(U_{V}^{\alpha} \times V\right) \cap S_{\alpha}\right): V \in \mathcal{V}_{n}, \alpha<\kappa\right\}
$$

has a $\sigma$-scattered network. We have

$$
g^{-1}\left(\left(U_{V}^{\alpha} \times V\right) \cap S_{\alpha}\right) \subset f^{-1}(V) \cap\left(U_{V}^{\alpha} \backslash \bigcup_{\beta<\alpha} U_{V}^{\beta}\right)
$$

for each $V \in \mathcal{V}_{n}$ and $\alpha<\kappa$. Now, if $\mathcal{N}_{n}$ is a $\sigma$-scattered network for $f^{-1}\left(\mathcal{V}_{n}\right)$ and $N \in \mathcal{N}_{n}$ is nonempty, there is a uniquely determined $V(N) \in \mathcal{V}_{n}$ such that $N \subset f^{-1}(V(N))$ as $\mathcal{V}_{n}$ is pairwise disjoint. Now the family $\left\{N \cap\left(U_{V(N)}^{\alpha} \backslash\right.\right.$ $\left.\left.\bigcup_{\beta<\alpha} U_{V(N)}^{\beta}\right): \alpha<\kappa\right\}$ is a scattered family of subsets of $N$ for every $N \in \mathcal{N}_{n}$ and so the family $\left\{N \cap\left(U_{V}^{\alpha} \backslash \bigcup_{\beta<\alpha} U_{V}^{\beta}\right): \alpha<\kappa, N \in \mathcal{N}_{n}\right\}$ is also $\sigma$-scattered and it is a network of

$$
\left\{f^{-1}(V) \cap\left(U_{V}^{\alpha} \backslash \bigcup_{\beta<\alpha} U_{V}^{\beta}\right): V \in \mathcal{V}_{n}, \alpha<\kappa\right\} .
$$

Since the latter family is pairwise disjoint, it is easy to observe that the family $\left\{g^{-1}\left(\left(U_{V}^{\alpha} \times V\right) \cap S_{\alpha}\right): V \in \mathcal{V}_{n}, \alpha<\kappa\right\}$ of respective subsets also has a $\sigma$-scattered network. 
(b) The graph of $f$ equals

$$
G=\{(x, y) \in X \times M: \varrho(f(x), y)=0\} .
$$

The map $g^{*}$ taking $(x, y)$ to $(x, y, f(x))$ is a particular case of the map $g$ from (a) with $X^{*}=X \times M$ in place of $X, f^{*}(x, y)=f(x)$ in place of $f$, and $\mathcal{A}^{*}$ in place of $\mathcal{A}$.

The projection map $p: X \times M \times M \rightarrow M \times M$ defined by $p(x, y, z)=$ $(y, z)$ is continuous, as is the metric $\varrho: M \times M \rightarrow \mathbb{R}$.

So the preimages of the open sets $\mathbb{R} \backslash\{0\}$ and $(-1 / k, 1 / k), k \in \mathbb{N}$, under $\varrho \circ p \circ g^{*}$ are in $\mathcal{A}^{*}$. As

$$
\begin{aligned}
G & =(X \times M) \backslash\left(\varrho \circ p \circ g^{*}\right)^{-1}(\mathbb{R} \backslash\{0\}) \\
& =\bigcap_{k \in \mathbb{N}}\left(\varrho \circ p \circ g^{*}\right)^{-1}(-1 / k, 1 / k),
\end{aligned}
$$

we conclude that $G$ is both in $\mathcal{A}_{c}^{*}$ and in $\mathcal{A}_{\delta}^{*}$.

3. Extensions of measurable maps and isomorphisms. Let $\mathcal{L}$ be a family of subsets of $X$ which contains the empty set. The family $\mathcal{S} \subset \mathcal{L}$ is called $\mathcal{L}$-hereditarily additive if $\bigcup \mathcal{T} \in \mathcal{L}$ whenever $\mathcal{T}=\left\{T_{S}: S \in \mathcal{S}\right\}$ with $T_{S} \subset S$ and $T_{S} \in \mathcal{L}$ for $S \in \mathcal{S}$.

Given $A \subset X$, we use $\mathcal{L}(A)$ to denote the family $\{L \cap A: L \in \mathcal{L}\}$. Note that $\mathcal{L}_{\sigma}(A)=(\mathcal{L}(A))_{\sigma}$.

The following proposition can be understood as an abstract form of the Kuratowski extension theorem on Borel measurable maps. It may be of some interest to note that if we limit ourselves just to the case of a separable complete metric space $M$, our proof gives a proof of the Kuratowski theorem by a simple reduction of the statements of the proposition to known results on extensions of continuous maps.

Proposition 3.1. Let $X$ be a nonempty set and $\mathcal{L}$ be an algebra of its subsets. Assume that $f: A \rightarrow M$ is an $\mathcal{L}_{\sigma}(A)$-measurable map of a subset $A$ of $X$ into a complete metric space $M$. Suppose there are $\mathcal{L}$-hereditarily additive and disjoint families $\mathcal{N}_{n}, n \in \mathbb{N}$, such that the family $\mathcal{N}=\bigcup_{n \in \mathbb{N}} \mathcal{N}_{n}(A)$ is a network for $f^{-1}(\mathcal{G}(M))$. Then there is a set $A^{*} \in \mathcal{L}_{\sigma \delta}$ with $A \subset A^{*}$ and an $\mathcal{L}_{\sigma}\left(A^{*}\right)$-measurable extension $f^{*}: A^{*} \rightarrow M$ of $f$. If the complement of $A$ belongs to $\mathcal{L}_{\sigma}$, then we may require that $A^{*}=X$.

Proof. We define $\mathcal{L}$-hereditarily additive partitions $\mathcal{N}_{n}^{*}=\mathcal{N}_{n} \cup\{X \backslash$ $\left.\bigcup \mathcal{N}_{n}\right\}$. For $x \in X$ put $q(x)=\left(N_{n} \in \mathcal{N}_{n}^{*}: x \in N_{n}\right) \in P=\prod_{n \in \mathbb{N}} \mathcal{N}_{n}^{*}$. We consider each partition $\mathcal{N}_{n}^{*}$ endowed with the discrete topology and the product space $P$ with the corresponding metrizable product topology. So it may be viewed as a completely metrizable subspace of the Baire space $B(\kappa)=\kappa^{\mathbb{N}}$ if $\kappa \geq \operatorname{card}\left(\mathcal{N}_{n}^{*}\right)$ for every $n \in \mathbb{N}$. 
For $G$ open in $P$, we have

$$
G=\bigcup_{k \in \mathbb{N}} \bigcup_{\left(N_{1}^{0}, \ldots, N_{k}^{0}\right) \in S_{k}}\left\{\left(N_{n}\right) \in P: N_{1}=N_{1}^{0}, \ldots, N_{k}=N_{k}^{0}\right\},
$$

where the union is taken over a set $S_{k}$ of $k$-tuples $\left(N_{1}^{0}, \ldots, N_{k}^{0}\right) \in \prod_{i=1}^{k} \mathcal{N}_{i}^{*}$, and so

$$
q^{-1}(G)=\bigcup_{k \in \mathbb{N}} \bigcup_{\left(N_{1}^{0}, \ldots, N_{k}^{0}\right) \in S_{k}} N_{1}^{0} \cap \cdots \cap N_{k}^{0} .
$$

By induction we now see that the families

$$
\left\{N_{1}^{0} \cap \cdots \cap N_{k}^{0}: N_{1}^{0} \in \mathcal{N}_{1}^{*}, \ldots, N_{k}^{0} \in \mathcal{N}_{k}^{*}\right\}
$$

are $\mathcal{L}$-hereditarily additive and so $\mathcal{L}$-additive.

Indeed, the family $\mathcal{N}_{1}^{*}$ is $\mathcal{L}$-hereditarily additive by our assumptions. Suppose the claim is true for $k \in \mathbb{N}$ and consider some sets $M\left(N_{1}^{0}, \ldots, N_{k+1}^{0}\right) \subset$ $N_{1}^{0} \cap \cdots \cap N_{k}^{0} \cap N_{k+1}^{0}$ from $\mathcal{L}$. Then for fixed $\left(N_{1}^{0}, \ldots, N_{k}^{0}\right) \in \prod_{i=1}^{k} \mathcal{N}_{i}^{*}$ each set

$$
M\left(N_{1}^{0}, \ldots, N_{k}^{0}\right)=\bigcup\left\{M\left(N_{1}^{0}, \ldots, N_{k}^{0}, N_{k+1}^{0}\right): N_{k+1}^{0} \in \mathcal{N}_{k+1}^{*}\right\}
$$

is a subset of $N_{1}^{0} \cap \cdots \cap N_{k}^{0}$. It belongs to $\mathcal{L}$ since $\mathcal{N}_{k+1}^{*}$ is $\mathcal{L}$-hereditarily additive. Now we use the induction hypothesis to get

$$
\bigcup\left\{M\left(N_{1}^{0}, \ldots, N_{k+1}^{0}\right):\left(N_{1}^{0}, \ldots, N_{k+1}^{0}\right) \in \prod_{i=1}^{k+1} \mathcal{N}_{i}^{*}\right\} \in \mathcal{L} .
$$

Thus the claim is proved. In particular, $q^{-1}(G)$ is in $\mathcal{L}_{\sigma}$ for every open $G$ and so $q$ is $\mathcal{L}_{\sigma}$-measurable.

Suppose that $f\left(x_{1}\right) \neq f\left(x_{2}\right)$ for some $x_{1}, x_{2} \in A$. This implies that there is an open set $U \subset M$ such that $f\left(x_{1}\right) \in U$ and $f\left(x_{2}\right) \notin U$. So there are $n \in \mathbb{N}$ and $N \in \mathcal{N}_{n}$ such that $x_{1} \in N \cap A \subset f^{-1}(U)$ and $x_{2} \in A \backslash(N \cap A)$. Thus $x_{1} \in N, x_{2} \notin N$ and so the sequences $q\left(x_{1}\right)$ and $q\left(x_{2}\right)$ differ in their $n$th coordinates.

So we may define $\varphi: B=q(A) \rightarrow M$ by $\varphi(y)=f(x)$ whenever $x \in$ $q^{-1}(y)$ and $y \in B$. Then $f=\varphi \circ q$ on $A$.

Let $G$ be any open subset of $M$. Then

$$
\varphi^{-1}(G)=q\left(f^{-1}(G)\right)=\bigcup\left\{q(N \cap A): N \in \mathcal{N}, N \cap A \subset f^{-1}(G)\right\}
$$

by the definition of $\varphi$ and since $\{N \cap A: N \in \mathcal{N}\}$ is a network for $f^{-1}(\mathcal{G}(M))$.

We may now observe that $q(N \backslash A) \cap q(A \backslash N) \subset q(N) \cap q(X \backslash N)=\emptyset$ and therefore

$$
q(N \cap A)=q(N) \cap q(A) \quad \text { for } N \in \mathcal{N} .
$$

By (1) and (2), $\varphi^{-1}(G)=B \cap \bigcup\left\{q(N): N \in \mathcal{N}, N \cap A \subset f^{-1}(G)\right\}$. As $q(N)=\left\{\left(N_{n}\right) \in q(X): N_{k}=N\right\}$ for $N \in \mathcal{N}_{k}$, the sets $q(N)$ are clopen 
in $q(X)$. This finally implies that $\varphi^{-1}(G)$ is an open set in $B$ and thus the map $\varphi: B \rightarrow M$ is continuous.

By $[9, \S 35$, I, Theorem 1], we may extend $\varphi: B \rightarrow M$ to a continuous map $\varphi^{*}: B^{*} \rightarrow M$ defined on a $\mathcal{G}_{\delta}$ set $B^{*} \subset P, B^{*} \supset B$.

As $q$ is $\mathcal{L}_{\sigma^{-}}$-measurable, $f^{*}=\varphi^{*} \circ q$ is an $\mathcal{L}_{\sigma}\left(A^{*}\right)$-measurable extension of $f$ defined on the set $A^{*}=q^{-1}\left(B^{*}\right)$ which belongs to $\mathcal{L}_{\sigma \delta}$.

If moreover $X \backslash A=\bigcup_{m \in \mathbb{N}} L_{m}$ with $L_{m} \in \mathcal{L}$, then we form a sequence $\mathcal{P}_{k}, k \in \mathbb{N}$, of $\mathcal{L}$-hereditarily additive partitions of $X$ consisting of all $\mathcal{N}_{n}^{*}$ 's and of all $\left\{L_{m}, X \backslash L_{m}\right\}, m, n \in \mathbb{N}$. Defining $q$ using the sequence $\left(\mathcal{P}_{k}\right)$ instead of $\left(\mathcal{N}_{n}^{*}\right)$, we arrive at the same situation as above, and moreover $q(X) \backslash q(A)$ is open in $q(X)$. So $q(A)$ is closed in the space $q(X)$ which has covering dimension 0 and the map $\varphi: B=q(A) \rightarrow M$ may be extended to a continuous map $\varphi^{*}: q(X) \rightarrow M$ (see, e.g., [11]). Hence $f^{*}=\varphi^{*} \circ q$ is defined on $X$, and the last statement of the proposition is proved.

To derive extension theorems for measurable maps of (scattered-) Borel classes, we formulate a topological version of the preceding result.

Proposition 3.2. Let $X$ be a topological space and $M$ be a completely metrizable space. Suppose that $\mathcal{M}$ is a family of subsets of $X$ such that

- $\mathcal{F} \cup \mathcal{G} \subset \mathcal{M}$,

- $\mathcal{M}$ is closed under finite intersections,

- $\mathcal{M}_{c} \subset \mathcal{M}_{\sigma}$.

Let $A \subset X$ and $f: A \rightarrow M$ be an $\mathcal{M}_{\sigma}(A)$-measurable map. If $M$ is nonseparable, assume further that $\mathcal{M}_{s}=\mathcal{M}$ and $f^{-1}(\mathcal{G}(M))$ has a $\sigma$-scattered network. Then there is an $A^{*} \supset A$ in $\mathcal{M}_{\sigma \delta}$ and an $\mathcal{M}_{\sigma}\left(A^{*}\right)$-measurable map $f^{*}: A^{*} \rightarrow M$ such that $f^{*} \mid A=f$. Moreover, if $X \backslash A \in \mathcal{M}_{\sigma}$, then we may require $A^{*}=X$.

Proof. We verify the assumptions of Proposition 3.1 with a suitable algebra $\mathcal{L}$.

Let $\mathcal{B}=\bigcup_{k \in \mathbb{N}} \mathcal{B}_{k}$ be an open base of the topology of $M$ such that each $\mathcal{B}_{k}$ is discrete (or contains at most one element if $M$ is separable).

By our assumptions $f^{-1}\left(\mathcal{B}_{k}\right)$ has a network $\mathcal{N}_{k}=\bigcup_{m \in \mathbb{N}} \mathcal{N}_{k, m}$, where the $\mathcal{N}_{k, m}$ are scattered if $M$ is nonseparable (they can be chosen to be singletons if $M$ is separable).

For each $N \in \mathcal{N}_{k, m}$ there is a single $B(N) \in \mathcal{B}_{k}$ such that $N \subset f^{-1}(B(N))$. Since $f^{-1}(B(N)) \in \mathcal{M}_{\sigma}(A)$, there are $M_{n}(N) \in \mathcal{M}$ such that $f^{-1}(B(N))=$ $\bigcup_{n \in \mathbb{N}} M_{n}(N) \cap A$.

There are sets $\widehat{N} \in \mathcal{F} \wedge \mathcal{G}$ such that $N \subset \widehat{N}$ for $N \in \mathcal{N}_{k, m}$ and the families $\left\{\widehat{N}: N \in \mathcal{N}_{k, m}\right\}$ are scattered. Put $\mathcal{N}_{k, m, n}^{*}=\left\{\widehat{N} \cap M_{n}(N): N \in \mathcal{N}_{k, m}\right\}$. Each $\mathcal{N}_{k, m, n}^{*}$ is scattered since $\left\{\widehat{N}: N \in \mathcal{N}_{k, m}\right\}$ is scattered. As each element 
of $\mathcal{N}_{k, m, n}^{*}$ belongs to $\mathcal{M}$, and $\mathcal{M}$ is closed under unions of scattered families (or $\mathcal{N}_{k, m, n}^{*}$ is a singleton if $M$ is separable), each $\mathcal{N}_{k, m, n}^{*}$ is $\mathcal{M}$-hereditarily additive. Since $\mathcal{M}_{c} \subset \mathcal{M}_{\sigma}$, each family $\mathcal{N}_{k, m, n}^{*}$ is $\mathcal{L}$-hereditarily additive, where $\mathcal{L}=\mathcal{M}_{\sigma} \cap \mathcal{M}_{\sigma c}$. As $\{\emptyset, X\} \subset \mathcal{F} \wedge \mathcal{G} \subset \mathcal{M}, \mathcal{L}$ contains the empty set. The family $\mathcal{L}$ is closed under complements by definition. Also, $\mathcal{L}$ is closed under finite intersections (and unions) since $\mathcal{M}$ is closed under finite intersections. So $\mathcal{L}$ is an algebra.

Finally, we have the inclusions

$$
\widehat{N} \cap M_{n}(N) \cap A \subset M_{n}(N) \cap A \subset f^{-1}(B(N)), \quad \bigcup_{n \in \mathbb{N}} M_{n}(N) \supset N
$$

which ensure that $\bigcup_{k, m, n \in \mathbb{N}} \mathcal{N}_{k, m, n}^{*}(A)$ is a $\sigma$-scattered network for $f^{-1}(\mathcal{B})$ and thus also for $f^{-1}(\mathcal{G}(M))$, and all the assumptions of Proposition 3.1 are verified.

As a corollary of the previous abstract results we get a theorem on the hierarchies of scattered-Borel sets:

Theorem 3.3. Let $A$ be a subset of a topological space $X, M$ be a complete metric space, and $f: A \rightarrow M$ be $\mathcal{A}_{\alpha}^{s}(A)$-measurable for an ordinal $\alpha \geq 1$. Assume further that

(i) $M$ is separable, or

(ii) $A$ is a Suslin subset of a complete metric space, or

(iii) $M$ has nonmeasurable weight and $A$ is an absolute Suslin $(\mathcal{H})$ space. Then there is an extension $f^{*}: A^{*} \rightarrow M$ of $f$ to $A^{*} \in \mathcal{M}_{\alpha+1}^{s}(X)$ such that $\left(f^{*}\right)^{-1}(\mathcal{G}(M)) \subset \mathcal{A}_{\alpha}^{s}\left(A^{*}\right)$. Moreover, if $X \backslash A \in \mathcal{A}_{\alpha}^{s}$, then we may require $A^{*}=X$.

Proof. Put $\mathcal{M}=\left(\bigcup_{\beta<\alpha} \mathcal{M}_{\beta}^{s}\right)_{s}$ for $\alpha>1$ and $\mathcal{M}=\mathcal{H}$ for $\alpha=1$.

Let us verify that $\mathcal{M}$ fulfils the assumption of Proposition 3.2. Clearly, $\mathcal{M}$ contains $\mathcal{F} \cup \mathcal{G}$. It is closed under finite intersections, because the family $\{A \cap B: A \in \mathcal{A}, B \in \mathcal{B}\}$ is scattered if both $\mathcal{A}$ and $\mathcal{B}$ are, and all the classes considered are closed under finite intersections by Lemma 2.1.

Finally, note that $\mathcal{M}_{\sigma}=\mathcal{A}_{\alpha}$ by the definition, and $\mathcal{M}_{c} \subset \mathcal{M}_{\sigma}$ by Lemma 2.1.

If $M$ is separable, Proposition 3.2 applies directly. So we may and will investigate the other cases only.

Let $\mathcal{B}=\bigcup_{k \in \mathbb{N}} \mathcal{B}_{k}$ be an open base for the topology of $M$ with $\mathcal{B}_{k}$ discrete.

Suppose that $A$ is Suslin in its metric completion. Then each $f^{-1}\left(\mathcal{B}_{k}\right)$ is disjoint and scattered-Borel-additive. As each scattered-Borel set in $A$ is Suslin in $A$, the family $f^{-1}\left(\mathcal{B}_{k}\right)$ is Suslin-additive, and using [1, Theorem 2] we find that it has a $\sigma$-discrete network $\mathcal{N}_{k}$. Hence $\mathcal{N}=\bigcup_{k \in \mathbb{N}} \mathcal{N}_{k}$ is a $\sigma$-discrete (and thus also $\sigma$-scattered) network of $f^{-1}(\mathcal{G}(M)$ ). 
If $A$ is an absolute $\mathcal{S}(\mathcal{H})$-space and the density of $M$ is nonmeasurable, then the cardinality of each $\mathcal{B}_{k}$ is nonmeasurable, and so is the cardinality of each $f^{-1}\left(\mathcal{B}_{k}\right)$. Being scattered-Borel-additive, the families $f^{-1}\left(\mathcal{B}_{k}\right)$ are $\operatorname{Suslin}(\mathcal{H})$-additive and disjoint, and thus by [7] they have a $\sigma$-scattered network.

Hence also in the other two cases Proposition 3.2 applies.

REMARK 3.4. The same holds if we replace $\mathcal{A}_{\alpha}^{s}$ and $\mathcal{M}_{\alpha+1}^{s}$ in the theorem by $(\mathcal{F} \wedge \mathcal{G})_{\alpha}$ and $(\mathcal{F} \wedge \mathcal{G})_{\alpha+1}$ for $\alpha$ odd and by $(\mathcal{F} \vee \mathcal{G})_{\alpha}$ and $(\mathcal{F} \vee \mathcal{G})_{\alpha+1}$ for $\alpha$ even, where the classes $(\mathcal{F} \wedge \mathcal{G})_{\alpha}$ and $(\mathcal{F} \vee \mathcal{G})_{\alpha}$ are those defined in [8]. Proposition 3.2 can then be applied to the family $\mathcal{M}=\left(\bigcup_{\beta<\alpha}(\mathcal{F} \vee \mathcal{G})_{\beta}\right)_{s}$ for $\alpha$ odd, and $\mathcal{M}=\left(\bigcup_{\beta<\alpha}(\mathcal{F} \wedge \mathcal{G})_{\beta}\right)_{s}$ for $\alpha \geq 2$ even. Here $\mathcal{F} \vee \mathcal{G}=\{F \cup G$ : $F \in \mathcal{F}, G \in \mathcal{G}\}$.

In the particular case of metrizable spaces, we get generalizations of the Kuratowski and Hansell results. The next theorems extend the results of Hansell [2, Theorems 9-11] as they include the case of limit classes. To get the following theorem from Theorem 3.3, we only use the fact that $\mathcal{A}_{\alpha}=\mathcal{A}_{\alpha}^{s}$ and $\mathcal{M}_{\alpha}=\mathcal{M}_{\alpha}^{s}$ for $\alpha<\omega_{1}$ in metric spaces.

Theorem 3.5. Let $f: A \rightarrow M$ be a Borel measurable map of class $\alpha<\omega_{1}$ of the Suslin subset $A$ of a complete metric space $X$ to the complete metric space $M$. Then there is an extension $f^{*}: A^{*} \rightarrow M$ that is Borel measurable of class $\alpha$ such that $A^{*}$ is of multiplicative class $\alpha+1$ in $X$. If $M$ is Polish, then $A$ can be an arbitrary subset of a topological space $X$.

Like in $[9, \S 35, \mathrm{VII}]$, as an immediate corollary we get a result on extension of isomorphisms:

TheOREm 3.6. Let $f: A \rightarrow B$ be a Borel isomorphism of class $(\alpha, \beta)$, $\alpha, \beta<\omega_{1}$, of a Suslin subset of some complete metric space $X$ onto a Suslin subset of some complete metric space $Y$. Then there is a Borel isomorphism $f^{*}: A^{*} \rightarrow B^{*}$ of class $(\alpha, \beta)$ that extends $f$ such that $A^{*}$ is Borel of multiplicative class $\alpha+\beta+1$ in $X$, and $B^{*}$ is Borel of multiplicative class $\beta+\alpha+1$ in $Y$.

Proof. Using Theorem 3.5, we get extensions $f^{\prime}: A^{\prime} \rightarrow Y$, with $A^{\prime}$ of multiplicative class $\alpha+1$, and $\left(f^{-1}\right)^{\prime}: B^{\prime} \rightarrow X$, with $B^{\prime}$ of multiplicative class $\beta+1$, of $f$ and $f^{-1}$, respectively. Consider the intersection of the graphs of $f^{\prime}$ and $\left(f^{-1}\right)^{\prime}$. By Lemma 2.4(b), it is of multiplicative class $\beta+1$ in the graph of $f^{\prime}$, and by Lemma 2.4(a), its projection $A^{*}$ to $X$ is of multiplicative class $\alpha+\beta+1$ in $A^{\prime}$. Hence $A^{*}$ is of multiplicative class $\alpha+\beta+1$ in $X$. Similarly, $B^{*}$ is of multiplicative class $\beta+\alpha+1$ in $Y$.

As another corollary of Theorem 3.5 we get an estimate on the class of the range of a Borel isomorphism of a given class. Let us remark that in the 
next section on Borel bimeasurable maps we get another proof of the special case of Borel isomorphisms of class $(0, \alpha)$ while we get a weaker result for Borel isomorphisms of the other classes.

TheOrem 3.7. Let $f: X \rightarrow Y$ be a Borel isomorphism of class $(\alpha, \beta)$, $\alpha, \beta<\omega_{1}$, of the absolute $\mathcal{M}_{\xi}, \xi \geq 1$, metrizable space $X$ onto the Suslin subset $Y$ of a complete metric space. Then $Y$ is an absolute $\mathcal{M}_{\beta+\max (\xi, \alpha)}$ space.

Proof. Let $\widehat{X}$ be a metric completion of $X$ and $g$ be the Borel measurable extension of class $\beta$ of $f^{-1}$ to an absolute $\mathcal{M}_{\beta+1}$ space $\widehat{Y}$, which exists by Theorem 3.5.

The graph of $f$ is of multiplicative class $\alpha$ in $X \times \widehat{Y}$ by Lemma 2.4(b), and so of multiplicative class $\max (\alpha, \xi)$ in $\widehat{X} \times \widehat{Y}$. The map $\widehat{g}: X \rightarrow \operatorname{graph}(g)$ defined by $\widehat{g}(x)=(x, g(x))$ is Borel measurable of class $\beta$ by Lemma 2.4(a), and so $Y=\widehat{g}^{-1}(\operatorname{graph}(f))$ is of multiplicative class $\beta+\max (\alpha, \xi)$ in $\widehat{Y}$ and, as $\xi \geq 1$, also in a compactification of $\widehat{Y}$. Using, e.g., [8, Theorems 2.5 and 3.8] this proves that $Y$ is of absolute class $\mathcal{M}_{\beta+\max (\xi, \alpha)}$.

REmARK 3.8. Applying Theorem 3.3 to extensions of scattered-Borel measurable isomorphisms of metric spaces as above, we obtain $A^{*}$ of class $\alpha+$ $1+\beta+1$ and $B^{*}$ of class $\beta+1+\alpha+1$ in general. Applying Theorem 3.3 to the estimate of the absolute class of the range of a scattered-Borel measurable map between metric spaces as above, we deduce that the range is of class $\beta+1+\max (\xi, \alpha+1)$ in general. Here we use part (a) of Lemma 2.3.

4. Ranges of bimeasurable maps. Let us say that a map $f: X \rightarrow Y$ is (scattered-) Borel bimeasurable of class $(\alpha, \beta)$ if $f$ is (scattered-) Borel measurable of class $\alpha$ and takes sets of additive, or multiplicative, class $\gamma$ to sets of the corresponding, additive or multiplicative, class $\beta+\gamma$. In fact, in some of the following statements, the assumption that $f$ is (scattered-) Borel measurable of class $\alpha$ is replaced by the, in general weaker, assumption that $f$ is $\mathcal{A}_{\alpha}^{s}$-measurable.

A Tikhonov topological space $X$ is absolute $\mathcal{M}_{\alpha}^{s}$ if it is in $\mathcal{M}_{\alpha}^{s}(Y)$ whenever it is embedded in another Tikhonov space $Y$. Notice that for metric spaces, belonging to an absolute class usually means to belong to the corresponding class in the metric completion which is an absolute $\mathcal{M}_{1}$ Tikhonov space. The key fact used in the proof of the following proposition is a characterization of absolute $\mathcal{M}_{\alpha}^{s}$ spaces in terms of complete sequences of covers. Let us therefore recall that a sequence of covers $\mathcal{C}_{n}, n \in \mathbb{N}$, of a topological space $X$ is complete if every filter $\mathcal{U}$ which intersects $\mathcal{C}_{n}$ for every $n \in \mathbb{N}$ has an accumulation point, i.e., $\bigcap\{\bar{U}: U \in \mathcal{U}\} \neq \emptyset$. As in [8] we use the fact that, in a regular Hausdorff space $X$, the sequence of covers $\mathcal{C}_{n}, n \in \mathbb{N}$, 
is complete if and only if given a (centered) sequence $C_{n} \in \mathcal{C}_{n}$ there is a (nonempty) compact set $K \subset X$ such that for every open $U \supset K$ we have $\overline{C_{1} \cap \cdots \cap C_{n}} \subset U$ for $n$ sufficiently large. The last property is abbreviated by saying that the sequence $\overline{C_{1} \cap \cdots \cap C_{n}}$ decreases to $K$.

Proposition 4.1. Let $X$ be an absolute $\mathcal{M}_{\xi}^{s}$ Tikhonov space with $\xi \geq 2$ or a completely metrizable space, and $Y$ be Suslin in a complete metric space $(M, \varrho)$. Let $f: X \rightarrow Y$ be a (scattered-) Borel bimeasurable surjection which is $\mathcal{A}_{\alpha}^{s}$-measurable and takes sets of additive, or multiplicative, class $\gamma$ to sets of the corresponding, additive or multiplicative, class $\beta+\gamma$. Suppose the family $f^{-1}(\mathcal{G}(Y))$ has a $\sigma$-scattered network. Then $Y$ is an absolute $\mathcal{M}_{\eta}^{s}$ space, where

(a) $\eta=\beta+\alpha+\max (\xi, \alpha)$ if $\alpha<\omega_{1}$,

(b) $\eta=\beta+\alpha+1+\max (\xi, \alpha+1)$ if $X$ is metrizable,

(c) $\eta=\beta+\alpha+3+\max (\xi, \alpha+1)$ in general.

Proof. If $X$ is an absolute $\mathcal{M}_{1}^{s}$ space and $f$ is a continuous open map, then $Y$ is absolute $\mathcal{M}_{1}^{s}$ by [8, Proposition 4.5]. For metric spaces absolute $\mathcal{M}_{1}^{s}$ coincides with complete metrizability and the particular case is a result of Hausdorff. So we may suppose further on that $\beta+\alpha+\xi \geq 2$.

We are going to reduce the study to the case of $\alpha=0$, i.e., to continuous bimeasurable maps only. So suppose first that $\alpha \geq 1$. The graph of $f$ is

$$
X^{*}=\{(x, y) \in X \times Y: \varrho(f(x), y)=0\} \in \mathcal{A}_{\alpha c}^{s} \cap \mathcal{M}_{\alpha+1}^{s}
$$

in $X \times M$ by Lemma 2.4(b) with $\mathcal{A}=\mathcal{A}_{\alpha}^{s}$. The family $\mathcal{A}^{*}$ is then a subfamily of $\mathcal{A}_{\alpha}^{s}(X \times M)$. Thus $X^{*}$ is of class $\mathcal{M}_{\alpha}^{s}(X \times M)$ in case (a), and it is of class $\mathcal{M}_{\alpha+1}^{s}(X \times M)$ in the other cases by Lemma 2.1.

Let $f^{*}$ denote the projection map of $X \times Y$ onto $Y$ restricted to $X^{*}$. We are going to show that it is a scattered-Borel bimeasurable map of $X^{*}$ onto $Y$ of a specified class. To do this we begin with some preliminary considerations.

Let $\mathcal{B}=\bigcup_{n \in \mathbb{N}} \mathcal{B}_{n}$ be a base of the topology of $Y$ such that $\mathcal{B}_{n}$ is discrete for $n \in \mathbb{N}$. Let $\mathcal{N}_{n}$ be a $\sigma$-scattered network of $f^{-1}\left(\mathcal{B}_{n}\right)$, which exists by our assumptions. By Lemma 2.2 we may find a $\sigma$-scattered refinement $\mathcal{N}_{n}^{*}$ of $f^{-1}\left(\mathcal{B}_{n}\right)$ consisting of elements of $\mathcal{A}_{\alpha}^{s}(X)$. As $f^{-1}\left(\mathcal{B}_{n}\right)$ is disjoint, $\mathcal{N}_{n}^{*}$ is a network of $f^{-1}\left(\mathcal{B}_{n}\right)$ and $\mathcal{N}^{*}=\bigcup_{n \in \mathbb{N}} \mathcal{N}_{n}^{*} \subset \mathcal{A}_{\alpha}^{s}$ is a $\sigma$-scattered network of $f^{-1}(\mathcal{G}(Y))$.

Define now a map $h$ by $h(x)=(x, f(x))$. By Lemmas 2.4(a) and 2.3, $h$ is a scattered-Borel measurable map of class $\alpha$ in case (a), of class $\alpha+1$ in case (b), and of class $\alpha+3$ in case (c).

Thus $f^{*}=f \circ h^{-1}$ maps sets of additive or multiplicative class $\gamma$ to sets of the corresponding, additive or multiplicative, class $\beta^{*}+\gamma$, where $\beta^{*}=\beta+\alpha$ in case (a), $\beta^{*}=\beta+\alpha+1$ in case (b), and $\beta^{*}=\beta+\alpha+3$ in case (c). 
So $f^{*}$ is a $\left(0, \beta^{*}\right)$-bimeasurable map of an absolute $\mathcal{M}_{\xi^{*}}^{s}$ space $X^{*}$ onto a Suslin subset $Y$ of the complete metric space $M$, where $\xi^{*}=\max (\xi, \alpha)$ in case (a) and $\xi^{*}=\max (\xi, \alpha+1)$ in the other two cases. Hence it is sufficient to prove the statement of the theorem for $\alpha=0$ and $\beta+\xi \geq 2$.

By [8, Theorem 3.8] or, e.g., by [10, Theorem 2.0] for the complete metric case, there is a complete sequence $\left(\mathcal{C}_{n}: n \in \mathbb{N}\right)$ of $\sigma$-scattered covers of $X$ and a nondecreasing sequence of ordinals $\xi_{n}<\xi$ such that $\mathcal{C}_{n} \subset \mathcal{A}_{\xi_{n}}^{s}(X)$ and $\beta+\xi_{n} \geq 1$.

We are going to find $\sigma$-scattered partitions $\mathcal{R}_{n}$ of $Y$ and maps $C_{n}: \mathcal{R}_{n} \rightarrow$ $\mathcal{C}_{n}$ such that $\mathcal{R}_{n} \subset \mathcal{A}_{\beta+\xi_{n}}^{s}$ in $Y, \mathcal{R}_{n+1}$ refines $\mathcal{R}_{n}$ and for $R_{n} \in \mathcal{R}_{n}$, and the unique sequence of $R_{i} \in \mathcal{R}_{i}, i=1, \ldots, n-1$, with $R_{1} \supset \cdots \supset R_{n}$, we have $R_{n} \subset f\left(\left(C_{1}\left(R_{1}\right) \cap \cdots \cap C_{n}\left(R_{n}\right)\right)\right.$.

We put $\mathcal{C}_{0}=\{X\}, \mathcal{R}_{0}=\{Y\}, C_{0}(Y)=X$ and proceed by induction over $n=0,1, \ldots$ Suppose $\mathcal{R}_{i}, C_{i}$ are already found for $i=0, \ldots, n$. Fix an $R_{n} \in \mathcal{R}_{n}$ (and thus also $R_{i} \in \mathcal{R}_{i}, i \leq n$, such that $R_{1} \supset \cdots \supset R_{n}$ ) and consider the $\sigma$-scattered family

$$
\mathcal{C}_{n+1}\left(R_{n}\right)=\left\{C_{0}\left(R_{0}\right) \cap \cdots \cap C_{n}\left(R_{n}\right) \cap C: C \in \mathcal{C}_{n+1}\right\} .
$$

Let $<$ be a well-ordering of $\mathcal{C}_{n+1}\left(R_{n}\right)$.

Define

$$
D_{n+1}(C)=f(C) \backslash \bigcup\left\{f\left(C^{\prime}\right): C^{\prime}<C, C^{\prime} \in \mathcal{C}_{n+1}\left(R_{n}\right)\right\}
$$

for $C \in \mathcal{C}_{n+1}\left(R_{n}\right)$ and note that $D_{n+1}(C)=f\left(C^{<}\right)$, where

$$
C^{<}=C \backslash f^{-1}\left(f\left(\bigcup\left\{C^{\prime}: C^{\prime}<C\right\}\right)\right) .
$$

Recall that $f$ is $(0, \beta)$-bimeasurable and $\mathcal{C}_{n+1}\left(R_{n}\right)$ is $\mathcal{A}_{\xi_{n+1}}^{s}$-additive in $X$. Thus $f^{-1}\left(f\left(\bigcup\left\{C^{\prime}: C^{\prime}<C\right\}\right)\right) \in \mathcal{A}_{\beta+\xi_{n+1}}^{s}$. As $C \in \mathcal{A}_{\xi_{n+1}}^{s}$ and $\mathcal{A}_{\xi_{n+1}}^{s} \cup$ $\left(\mathcal{A}_{\beta+\xi_{n+1}}^{s}\right)_{c} \subset \mathcal{M}_{\beta+\xi_{n+1}+1}^{s} \subset \mathcal{A}_{\beta+\xi_{n+1}+2}^{s}$ by Lemma 2.1, we have $C^{<} \in$ $\mathcal{A}_{\beta+\xi_{n+1}+2}^{s}$. The family $\mathcal{C}^{<}\left(R_{n}\right)=\left\{C^{<}: C \in \mathcal{C}_{n+1}\left(R_{n}\right)\right\}$ is $\sigma$-scattered and so $\mathcal{A}_{\beta+\xi_{n+1}+2}^{s}$-additive. By our assumptions

$$
\mathcal{D}_{n+1}\left(R_{n}\right)=\left\{D_{n+1}(C): C \in \mathcal{C}_{n+1}\left(R_{n}\right)\right\}=f\left(\mathcal{C}_{n+1}^{<}\left(R_{n}\right)\right) \subset \mathcal{A}_{\beta+\beta+\xi_{n+1}+2}^{s}(Y)
$$

and so it is a disjoint Suslin-additive family in $M$. By [1, Theorem 2] there is a $\sigma$-discrete refinement $\mathcal{E}_{n+1}\left(R_{n}\right)$ of $\mathcal{D}_{n+1}\left(R_{n}\right)$. Consequently, $\mathcal{E}_{n+1}\left(R_{n}\right)$ is a $\sigma$-discrete refinement of $f\left(\mathcal{C}_{n+1}\left(R_{n}\right)\right)$.

For every $E \in \mathcal{E}_{n+1}\left(R_{n}\right)$ choose a $C(E) \in \mathcal{C}_{n+1}\left(R_{n}\right)$ such that $E \subset$ $f(C(E))$. As $f(C(E)) \in \mathcal{A}_{\beta+\xi_{n+1}}^{s}(Y)$, there is a $\sigma$-scattered family $\left\{E_{a}: a \in\right.$ $A(E)\} \subset \mathcal{H} \cup \bigcup_{\gamma<\beta+\xi_{n+1}} \mathcal{M}_{\gamma}^{s}$ in $Y\left(\right.$ as $\left.\beta+\xi_{n+1} \geq 1\right)$ such that $f(C(E))=$ $\bigcup\left\{E_{a}: a \in A(E)\right\}$. Thus

$$
\mathcal{T}_{n+1}\left(R_{n}\right)=\left\{E_{a} \cap \bar{E}: E \in \mathcal{E}_{n+1}\left(R_{n}\right), a \in A(E)\right\}
$$


is a $\sigma$-scattered refinement of the family $f\left(\mathcal{C}_{n+1}\left(R_{n}\right)\right)$ with elements in $\mathcal{H} \cup$ $\bigcup_{\gamma<\beta+\xi_{n+1}} \mathcal{M}_{\gamma}^{s}$. Using now, e.g., [8, Lemma 3.2(b)], we infer that there is a pairwise disjoint $\sigma$-scattered family $\mathcal{R}_{n+1}\left(R_{n}\right)$ which refines $\mathcal{T}_{n+1}\left(R_{n}\right)$ and consists of elements of $\mathcal{H} \cup \bigcup_{\gamma<\beta+\xi_{n+1}} \mathcal{M}_{\gamma}^{s}$ (note that $\mathcal{M}_{0}^{s}=\mathcal{H}$ in [8]). Lemma 2.1 now ensures that $\mathcal{R}_{n+1}\left(R_{n}\right) \subset \mathcal{A}_{\beta+\xi_{n+1}}^{s}(Y)$.

Put $\mathcal{R}_{n+1}=\bigcup\left\{R_{n+1} \cap R_{n}: R_{n+1} \in \mathcal{R}_{n+1}\left(R_{n}\right), R_{n} \in \mathcal{R}_{n}\right\}$. Obviously, $\mathcal{R}_{n+1} \subset \mathcal{A}_{\beta+\xi_{n+1}}^{s}(Y)$ since $R_{n} \in \mathcal{A}_{\beta+\xi_{n}}^{s}(Y)$ and $\xi_{n} \leq \xi_{n+1}$. As each $\left\{R_{n} \cap\right.$ $\left.R_{n+1}: R_{n+1} \in \mathcal{R}_{n+1}\left(R_{n}\right)\right\}$ is a $\sigma$-scattered partition of $R_{n}, \mathcal{R}_{n+1}$ is a $\sigma$ scattered partition of $Y$. For every $R_{n+1} \in \mathcal{R}_{n+1}$ we choose a $C_{n+1} \in \mathcal{C}_{n+1}$ such that $R_{n+1} \subset f\left(C_{1}\left(R_{1}\right) \cap \cdots \cap C_{n+1}\left(R_{n+1}\right)\right)$, where $R_{i} \in \mathcal{R}_{i}$ for $i=$ $1, \ldots, n$ and $R_{1} \supset \cdots \supset R_{n+1}$.

Finally, we show that the sequence $\left(\mathcal{R}_{n}: n \in \mathbb{N}\right)$ is complete. Let $R_{n} \in$ $\mathcal{R}_{n}$ be a centered sequence. Let $C_{n}=C_{n}\left(R_{n}\right)$. By the properties of the maps $C_{n}$, the sequence of sets $C_{n}$ is centered. By the completeness of $\left(\mathcal{C}_{n}\right.$ : $n \in \mathbb{N}$ ), the sequence of sets $\overline{C_{1} \cap \cdots \cap C_{n}}, n \in \mathbb{N}$, decreases to a (nonempty) compact set $K$. As $f$ is continuous, the sequence $f\left(\overline{C_{1} \cap \cdots \cap C_{n}}\right), n \in \mathbb{N}$, decreases to the (nonempty) compact set $f(K)$. Using the regularity of $Y$, we see that also $\left(\overline{f\left(C_{1} \cap \cdots \cap C_{n}\right)}: n \in \mathbb{N}\right)$ decreases to $f(K)$ and the sequence of covers $\mathcal{R}_{n}$ is complete.

REMARK 4.2. If $X$ is metrizable, $\xi=2$ and $f$ is bimeasurable of class $(1,0)$, then we find that $f(X)$ is absolute $\mathcal{M}_{3}^{s}$. The Kuratowski and Hansell theorems give lower classes of the range if $f$ is a Borel isomorphism in this and other cases with $\alpha>0$.

REMARK 4.3. We did not use the existence of the $\sigma$-scattered network of $f^{-1}(\mathcal{G}(Y))$ in the case $\alpha=0$, but our assumption did not weaken the theorem because the disjoint families $f^{-1}\left(\mathcal{B}_{n}\right)$ are open and thus also scattered in this case. (The notation $\mathcal{B}_{n}$ was introduced in the above proof.)

Finally, we formulate the corollaries concerning images of a metric space $X$ and of a topological space $X$ of given absolute multiplicative classes.

TheOREM 4.4. Let $f: X \rightarrow Y$ be a scattered-Borel bimeasurable surjection of class $(\alpha, \beta)$ for some $\alpha, \beta \geq 0$ and let $Y$ be an absolute Suslin metric space. If $X$ is an absolute $\mathcal{M}_{\xi}^{s}$ metric space for some $\xi \geq 1$, then $Y$ is absolute $\mathcal{M}_{\eta}^{s}$, where $\eta=\beta+\alpha+\max (\xi, \alpha)$ if $\alpha<\omega_{1}$ and $\eta=\beta+\alpha+1+\max (\xi, \alpha+1)$ if $\alpha \geq \omega_{1}$.

Proof. Since $X$ is an absolute $\mathcal{M}_{\xi}^{s}$ metric space, it is absolute Suslin. The family $f^{-1}(\mathcal{G}(Y))$ has a $\sigma$-discrete network by the existence of a $\sigma$-discrete base in $Y$ and the Hansell theorem [1, Theorem 2] for preimages of its elements. Now we may apply Proposition 4.1. 
Let us remark that if $X$ is separable, then $Y$ is analytic and so absolute Suslin automatically in the situation of the previous theorem.

Theorem 4.5. Let $f: X \rightarrow Y$ be a scattered-Borel bimeasurable surjection of class $(\alpha, \beta)$ for some $\alpha, \beta \geq 0$ and suppose $Y$ is an absolute Suslin metric space with nonmeasurable density. If $X$ is an absolute $\mathcal{M}_{\xi}^{s}$ space, $\xi \geq 2$, then $Y$ is an absolute $\mathcal{M}_{\eta}^{s}$ metric space, where $\eta=\beta+\alpha+\max (\xi, \alpha)$ if $\alpha<\omega_{1}$, and $\eta=\beta+\alpha+3+\max (\xi, \alpha+1)$ in general.

Proof. We proceed as in the previous proof. We only use the fact that scattered-Borel sets are in $\mathcal{S}(\mathcal{H})$ and the theorem of [7, Theorem 2(a)] on $\mathcal{S}(\mathcal{H})$-additive families instead of [1, Theorem 2].

The author would like to express his gratitude to his colleagues Jiří Spurný and Miroslav Zelený for many useful comments.

\section{References}

[1] R. W. Hansell, Borel measurable mappings for nonseparable metric spaces, Trans. Amer. Math. Soc. 161 (1971), 145-169.

[2] -, On Borel mappings and Baire functions, ibid. 194 (1974), 195-211.

[3] - On characterizing non-separable analytic and extended Borel sets as types of continuous images, Proc. London Math. Soc. 28 (1974), 683-699.

[4] -, Descriptive topology, in: M. Hušek and J. van Mill (eds.), Recent Progress in General Topology, North-Holland, Amsterdam, 1992, 275-315.

[5] -, Descriptive sets and the topology of nonseparable Banach spaces, Serdica Math. J. 27 (2001), 1-66.

[6] P. Holický, Čech analytic and almost K-descriptive spaces, Czechoslovak Math. J. 43 (118) (1993), 451-466.

[7] -, Borel maps with the "point of continuity property" and completely Borel additive families in some nonmetrizable spaces, Proc. Amer. Math. Soc. 120 (1994), 951-958.

[8] P. Holický and J. Pelant, Internal descriptions of absolute Borel classes, to appear.

[9] K. Kuratowski, Topology, Vol. I, Academic Press, New York, 1966.

[10] W. Marciszewski and J. Pelant, Absolute Borel classes and function spaces, Trans. Amer. Math. Soc. 349 (1997), 3585-3596.

[11] E. Michael, Some refinements of a selection theorem with 0-dimensional domain, Fund. Math. 140 (1992), 279-287.

Petr Holický

Department of Mathematical Analysis

Charles University

Sokolovská 83

18600 Praha 8, Czech Republic

E-mail: holicky@karlin.mff.cuni.cz

Received February 6, 2004;

received in final form April 20, 2004 